\title{
Utilização do eugenol em Jundiá da Amazônia (Leiarius marmoratus): implicações na sedação e avaliação hemogasométrica
}

\section{Use of eugenol in Jundiá da Amazônia (Leiarius marmoratus): effects on sedation and evaluation hemogasometry}

\author{
Claucia Aparecida Honorato ${ }^{1 *}$; Andre Dambros²; Vanessa Menegatti Marcondes ${ }^{3}$; \\ Camila Aparecida Nascimento ${ }^{1}$
}

\begin{abstract}
Resumo
Este trabalho teve como finalidade avaliar o eugenol como anestésico para jundiá da Amazônia (Leiarius marmoratus). Foram mensurados o tempo de indução e recuperação à anestesia em diferentes concentrações de eugenol e seus efeitos nas trocas gasosas. Os resultados foram analisados segundo um delineamento inteiramente casualisado (DIC) com oito tratamentos (controle, 10, 20, 40, 80, 120, 150, $200 \mathrm{mg} \mathrm{L}^{-1}$ de eugenol) e dez repetições cada. Os valores de tempo de indução à anestesia e recuperação foram submetidos à regressão polinomial $(\mathrm{P}>0,05)$. As análises de variância (ANOVA) e as médias foram comparadas pelo teste de Tukey. Os parâmetros sanguíneos analisados foram glicose, $\mathrm{pH}$, pressão parcial de oxigênio $\left(\mathrm{PaO}_{2}\right)$, pressão parcial de dióxido de carbono $\left(\mathrm{PaCO}_{2}\right)$, concentração de bicarbonato $\left(\mathrm{HCO}_{3}^{-}\right)$, Sódio $(\mathrm{Na})$, Potássio $(\mathrm{K})$, Cálcio $(\mathrm{Ca})$ e Cloreto $(\mathrm{Cl})$. As concentrações de 40 e $80 \mathrm{mg} . \mathrm{L}^{-1}$

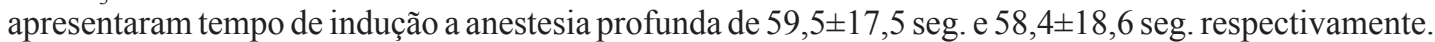
As concentrações acima de $120 \mathrm{mg}$. L- ${ }^{-1}$ apresentaram mortalidade. Estes peixes submetidos à diferentes concentrações de eugenol não apresentaram alterações na pressão de oxigể nio no sangue $\left(\mathrm{PaO}_{2}\right)$ e pressão de dióxido de carbono $\left(\mathrm{PaCO}_{2}\right)$. Os peixes quando submetidos a concentrações acima de $80 \mathrm{mg}$. $\mathrm{L}^{-1}$ apresentaram aumento de bicarbonato. Os valores de glicose plasmática apresentaram elevação significativa em resposta ao banho anestésico com eugenol. As concentrações de sódio, cloreto, potássio e proteína plasmática mantiveram-se constantes indicando que o processo de anestesia não foi duradouro o suficiente para induzir alterações no balanço eletrolítico desta espécie. Foi possível concluir que o eugenol é um anestésico adequado para o jundiá da Amazônia.
\end{abstract}

Palavras-chave: Manejo de peixes, anestesia, hemogasometria

\begin{abstract}
This work aims to evaluate the use of eugenol as an anesthetic for jundiá da Amazônia (Leiarius marmoratus), measuring the time to anesthesia induction in different concentrações and their effects in gas exchange. The results were analyzed according to a completely randomized design (DIC) with eight treatments (control, 10, 20, 40, 80, 120, 150, $200 \mathrm{mg} \mathrm{L}^{-1}$ of eugenol) and ten repetitions. Time values of anesthetic induction and recovery, according to the level of eugenol underwent polynomial regression $(\mathrm{p}<0.05)$. The analysis of variance (ANOVA) and averages were compared by Tukey test. Were determined the time of anesthesia and recovery. The parameters were analyzed blood glucose, $\mathrm{pH}$, partial pressure of oxygen $(\mathrm{PaO} 2)$, partial pressure of carbon dioxide $(\mathrm{PaCO} 2)$, concentration of
\end{abstract}

\footnotetext{
${ }^{1}$ Prof $^{\text {as }}$, Hospital Veterinário, Faculdade de Ciências Biológicas e da Saúde, Medicina Veterinária, Centro Universitário da Grande Dourados, UNIGRAN, Dourados, MS. E-mail: clauciahonorato@yahoo.com.br; camila.nascimento05@gamil.com

${ }^{2}$ Veterinário da Piscicultura Douradense Ltda, Dourados, MS. E-mail: andredambros@hotmail.com

${ }^{3}$ Prof $^{a}$ de Biomedicina, UNIGRAN, Dourados, MS. E-mail: vanessamarcondes@unigran.br

* Autor para correspondência
} 
bicarbonate $\left(\mathrm{HCO}_{3}^{-}\right)$, sodium $(\mathrm{Na})$, potassium $(\mathrm{K})$, calcium $(\mathrm{Ca})$ and chloride $(\mathrm{Cl})$. The concentrations of 40 and $80 \mathrm{mg} \cdot \mathrm{L}^{-1}$ of eugenol were the ones who presented time deep anesthesia induction of $59.5 \pm$ $17.5 \mathrm{sec}$. and $58.4 \pm 18.6 \mathrm{sec}$. respectively. The concentrations above $120 \mathrm{mg}$. $\mathrm{L}^{-1}$ presented mortality. These fish subjected to different level of eugenol did not show changes in blood oxygen pressure $\left(\mathrm{PaO}_{2}\right)$ and carbon dioxide $\left(\mathrm{PaCO}_{2}\right)$. The fish when subjected to concentrations above $80 \mathrm{mg} \mathrm{L}^{-1}$ showed an increase of bicarbonate. The plasma glucose values showed significant elevation in response to the bath with anesthetic eugenol. The concentrations of sodium, chloride, potassium and plasma protein remained constants indicating that the process of anesthesia was not long lasting enough to induce changes in the electrolyte balance. Within the conditions of this study, it can be concluded that eugenol is a suitable anesthetic for the silver. This product may be used at a concentration of $40 \mathrm{mg}$. $\mathrm{L}^{-1}$ without causing changes hemogasométrica.

Key words: Fish management, anesthesia, hemogasometry

\section{Introdução}

A piscicultura é uma atividade em crescimento no Brasil e dentre as espécies com potencial está o jundiá da Amazônia (Leiarius marmoratus) que vem se destacando no cenário de peixes de couro, devido seu habito alimentar onívoro, e característica organolépticas favoráveis de sua carne branca (SÁNCHEZ; MOYETONES; CERDÁ, 2009).

Dentro da piscicultura as práticas de manejo de são inevitáveis, por razões operacionais intrínsecas a atividade aquícola. Estas são associadas a fatores ambientais são as principais causas de estresse para os peixes (ZAHL; SAMUELSEN; KIESSLING, 2012). Os peixes quando são submetidos à captura, tendem a fuga passam por posterior exposição aérea, resultando entre outras respostas, no aumento da concentração de lactato e íons $\mathrm{H}^{+}$no músculo, que podem ser identificados na corrente sanguínea, além de elevadas concentrações de cortisol e glicose (TAHMASEBI-KOHYANI et al., 2012).

Diversas estratégias são apontadas na literatura com a finalidade de atenuar os efeitos do estresse em peixes, dentre eles a utilização de anestésico em algumas práticas de manejo (INOUE; HACKBARTH; MORAES, 2004). De acordo com Gimbo et al. (2008), os anestésicos são muito utilizados na piscicultura intensiva para diminuir a mortalidade dos peixes e facilitar o manejo. Como cada anestésico exige concentração diferente para induzir o estágio anestésico desejado, é necessário testar várias concentrações antes do tratamento definitivo, para não causar mortalidade dos animais expostos ao fármaco (ZAHL; SAMUELSEN; KIESSLING, 2012).

Dentre os anestésicos com potencial para utilização na piscicultura destaca-se o eugenol. O cravo da Índia possui alto percentual de eugenol. Esta substancia é muito usado na odontologia como componente de seladores e outros produtos anestésico de higiene bucal, tendo comprovado efeito bactericida e também tem sido empregado para a produção de outros compostos fenólicos (MAZZAFERA, 2003). O eugenol tem sido recomendado como um anestésico alternativo para peixes por ser um produto natural e aparentemente com poucos riscos de intoxicação (INOUE et al., 2011). Devido às características de eficácia, seguridade edebaixo custo(INOUE; HACKBARTH; MORAES, 2004) e já foi demonstrado sua eficácia para matrinxã Brycon cephalus (INOUE; SANTOS NETO; MORAES, 2003), tambaqui Colossoma macropomum (INOUE et al., 2011) tilápia Oreochromis niloticus (DELBON; PAIVA, 2012), pacu Piaractus mesopotamicus (ROTILI et al., 2012), pampo Trachinotus marginatus (OKAMOTO et al., 2009).

A anestesia em peixes pode ser afetada por fatores biológicos tais como diferença entre espécies e de tamanho (ROTILI et al., 2012). Dentre os estágios de anestesia de peixe observase nos primeiros estágios (I e II) a diminuição dos batimentos operculares e no estágio III aumento 
dos movimentos operculares como descrito por Ross; Ross (2008). Como as brânquias dos peixes tem múltiplas finalidades, desde promover as trocas gasosas, regulação osmótica iônica, regulação na excreção do nitrogênio (EVANS; PIERMARINI; CHOE, 2005) e hematológicas (KANANI; AHADIZADEH, 2013) a diminuição nos movimentos operculares podem alterar estas funções. A utilização de anestésicos pode causar reações adversas secundárias tais como acidose e estresse osmótico devido a parada respiratória e insuficiente troca gasosa e iônica entre o sangue e a água (ZAHL; SAMUELSEN; KIESSLING, 2012).

Este trabalho tem como finalidade avaliar o uso do eugenol como anestésico para jundiá da Amazônia, mensurando o tempo de indução à anestesia em relação a diferentes concentrações e seus efeitos nas trocas gasosas.

\section{Material e Métodos}

Esse trabalho foi desenvolvido no Hospital Veterinário do Centro Universitário da Grande Dourados (UNIGRAN). Os alevinos de jundiá da Amazônia foram adquiridos na Piscicultura Douradense localizada em Dourados - Mato Grosso do Sul.

Ospeixes forammantidosemumtanquesde $1.000 \mathrm{~L}$ de água termostatizada e aerada. Nessas condições os peixes passaram por sete dias de aclimatação às novas condições. Os peixes foram alimentados com ração comercial ad libtum (45\%Proteína bruta 3800 kcal. $\mathrm{Kg}^{-1}$ Energia digestível). A qualidade de água foi monitorada diariamente com o multiparâmetro HANNA modelo HI929828-13, no qual foi aferido: temperatura, potencial hidrogeniônico $(\mathrm{pH})$ e oxigênio dissolvido.A água foi mantida em condições semelhantes entre a caixa de aclimatação dos peixes, a unidade de anestesia e a unidade de recuperação à anestesia, registrando-se temperatura média de $22,0 \pm 0,3^{\circ} \mathrm{C}, \mathrm{pH}$ de $6,8 \pm 0,2$ e oxigênio dissolvido 4,8 $\pm 0,4 \mathrm{mg} . \mathrm{L}^{-1}$, intervalos considerados adequados para a manutenção de espécies de peixes tropicais (URBINATI; GONÇALVES, 2005).

O eugenol por ser oleoso, foi diluído em álcool etílico $\left(92,8^{\circ}\right)$ no momento do uso, no que resultou em solução-estoque à concentração de 100 $\mathrm{mg} \mathrm{mL}^{-1}(1: 10)$. Para se determinar a influência da concentração, na indução e na recuperação anestésica, foram testados oito tratamentos (controle, 10, 20, 40, 80, 120, 150, 200mg L-1 de eugenol). Para cada tratamento, foram utilizados dez juvenis $(n=10)$, coletados aleatoriamente e submetidos, um de cada vez, ao banho anestésico.

O ensaio foi conduzido com 80 juvenis de jundiá da Amazônia com peso médio de 83,93 $\pm 21,85 \mathrm{~g}$. O tempo de anestesia, bem como o tempo de recuperação, foi obtido por meio de um cronômetro digital. O tempo necessário para a latência total dos peixes, considerado o período entre o início da exposição até a perda total de equilíbrio na coluna de água e a parada dos batimentos operculares, condição equivalente ao estágio III de anestesia de acordo com Ross e Ross (2008). Após atingir o estágio III, os peixes foram pesados, retirouse o sangue e posteriormente transferidos para um aquário com água sem anestésico, onde foi monitorado o tempo de recuperação, considerado como o período necessário para que os peixes recuperassem o equilíbrio e a natação ativa na coluna de água.

A coleta de sangue foi realizada com seringa de três $\mathrm{mL}$ heparinizada pela veia caudal e subsequentemente analisada em aparelho de hemogasometria (Cobas HB121 - Roche Diagnostica Brasil, São Paulo, SP, Brasil). Os parâmetros sanguíneos analisados foram $\mathrm{pH}$, pressão parcial de oxigênio $\left(\mathrm{PaO}_{2}\right)$, pressão parcial de dióxido de carbono $\left(\mathrm{PaCO}_{2}\right)$, concentração de bicarbonato $\left(\mathrm{HCO}_{3}^{-}\right)$, Sódio $(\mathrm{Na})$, Potássio $(\mathrm{K})$, Cálcio $(\mathrm{Ca})$, Cloreto (Cl) e glicose (TRINDER, 1969).

Os resultados foram analisados segundo um delineamento inteiramente casualisado (DIC) com oito tratamentos e dez repetições. Os valores de tempo de indução anestésica e recuperação, de 
acordo com o nível de eugenol foram submetidos à regressão polinomial $(\mathrm{P}>0,05)$. As análises de variância (ANOVA) e as médias foram comparadas pelo testes de Tukey.

\section{Resultados e Discussão}

Em todas as concentrações, os animais atingiram o estágio de anestesia profunda em diferentes tempos (Tabela 1). Durante a indução anestésica, o comportamento dos peixes seguiu o padrão: hiperatividade ao primeiro contato com o anestésico, caracterizada pela natação agitada contra as paredes do aquário; natação lenta; diminuição dos batimentos operculares, parada do animal na posição dorso ventral ("barriga para cima"), perda da reação aos estímulos externos; perda do tônus muscular. Concentrações acima de 120mg. $\mathrm{L}^{-1}$ apresentaram mortalidade.

Tabela 1. Tempo de indução e recuperação e taxa de mortalidade do jundiá da Amazônia (Leiarius marmoratus) submetidos a diferentes concentrações de eugenol.

\begin{tabular}{|c|c|c|c|}
\hline $\begin{array}{c}\text { Concentração } \\
\left(\text { mg.L.' }{ }^{-1}\right)\end{array}$ & $\begin{array}{c}\text { Indução } \\
\text { (seg.) }\end{array}$ & $\begin{array}{l}\text { Recuperação } \\
\text { (seg.) }\end{array}$ & $\begin{array}{c}\text { Taxa de mortalidade } \\
(\%)^{*}\end{array}$ \\
\hline 10 & $237,00 \pm 29,34 a$ & $188,52 \pm 17,12 \mathrm{c}$ & 0 \\
\hline 20 & $176,64 \pm 53,18 b$ & $275,50 \pm 17,10 b$ & 0 \\
\hline 40 & $59,50 \pm 17,54 \mathrm{c}$ & $271,80 \pm 4,12 b$ & 0 \\
\hline 80 & $58,40 \pm 18,65 \mathrm{c}$ & $261,30 \pm 18,88 \mathrm{ab}$ & 0 \\
\hline 120 & $43,40 \pm 16,62 c$ & $350,88 \pm 16,00 \mathrm{a}$ & 10 \\
\hline 150 & $42,00 \pm 16,27 \mathrm{c}$ & $504,90 \pm 17,23 a$ & 20 \\
\hline 200 & $37,10 \pm 16,83 \mathrm{c}$ & $515,00 \pm 15,74 \mathrm{a}$ & 20 \\
\hline
\end{tabular}

Letras distintas reportam diferença estatística pelo teste de Tukey $(\mathrm{P}>0,05)$. * Número de peixes mortos durante a indução anestésica. Fonte: Elaboração dos autores.

As concentrações de 40 e $80 \mathrm{mg} . \mathrm{L}^{-1}$ de eugenol foram os que apresentaram tempo de indução a anestesia profunda de $59,5 \pm 17,5 \mathrm{seg}$. e $58,4 \pm 18,6$ seg., respectivamente sem causar alterações nos parâmetros avaliados. Estes resultados são semelhantes aos observados para juvenis de matrinxã (Brycon amazonicus), com peso aproximado de $50 \mathrm{~g}$ quando submetidos às concentrações de 50 e $75 \mathrm{mg}$. $\mathrm{L}^{-1}$ e atingiram anestesia profunda (INOUE; SANTOS NETO; MORAES, 2003). A concentração adequada de eugenol para indução e recuperação anestésica em jundiás (Rhamdia voulezi) com peso variando de 32,5 a 450 g é de 50 mg. $1^{-1}$ (DIEMER et al., 2012). Pereira-da-Silva et al. (2009) observou dose e tempo semelhante indução à anestesia profunda com 40 e $60 \mathrm{mg} \mathrm{L}^{-1}$ de óleo de cravo, no entanto, sugerem maior resistência dos alevinos de lambari (Astyanax bimaculatus) devido sua diferença de peso em comparação as demais espécies. Para jundiá da Amazônia (Leiarius marmoratus) a concentração de $40 \mathrm{mg} . \mathrm{L}^{-1}$ de eugenol foi eficiente em promover diminuição da atividade de natação, possibilitando o manejo eficaz destes. A utilização de baixa concentração de substancias anestésica é útil economicamente, possibilitando a adoção deste fármaco.

A dose de $200 \mathrm{mg}$. $\mathrm{L}^{-1}$ de eugenol proporcionou anestesia rápida dos peixes $(37,10 \pm 16,83$ segundos $)$, no entanto, o tempo de recuperação foi de $515 \pm 57,44$ seg. O tempo de recuperação aceitável e seguro após indução à anestesia profunda deve ser inferior a 600 segundos (SON et al., 2001 e PARK et al., 2008). O menor tempo de recuperação do jundiá da Amazônia (Leiarius marmoratus) à anestesia 
profunda foi observado para as concentrações de $10 \mathrm{mg} \mathrm{L-}{ }^{1}$. Observa-se que o tempo de recuperação na dose de $40 \mathrm{mg}$. L-1 ${ }^{1}$ foi de 278,1 seg. De acordo com Pereira-da-Silva et al. (2009), a indução à anestesia profunda deve levar de 1 a 3 min., e a recuperação não deve ultrapassar $5 \mathrm{~min}$. Okamoto et al. (2009) descreveu para pampo Trachinotus marginatus que ao expor à concentração mais baixa de eugenol (25 mg. $\left.\mathrm{l}^{-1}\right)$ levaram mais tempo para atingir a latência total (aproximadamente $10 \mathrm{~min})$ do que na maior concentração $\left(75 \mathrm{mg} . \mathrm{l}^{-1}\right)$, cujo tempo foi de $2 \mathrm{~min}$ que estas são inversamente proporcional ao tempo de recuperação.

A curva de regressão obtida em relação à indução anestésica (Figura 1A) apresentou queda elevada no tempo de indução, nas primeiras concentrações, com tendência à estabilidade nas últimas, seguindo o mesmo modelo matemático encontrado para Brycon amazonicus (INOUE; HACKBARTH; MORAES, 2004), para Oreochromis niloticus (VIDAL et al., 2008), para Salminus brasiliensis (PÁDUA et al., 2010). A curva de recuperação mostrou estabilidade entre as concentrações de 20 a $80 \mathrm{mg}$ de eugenol. $\mathrm{L}^{-1}$ (Figura 1B).

Figura 1. Tempo de indução (a) e de recuperação (B) de jundiá da Amazônia (Leiarius marmoratus) submetidos à diferentes concentrações de eugenol.

A

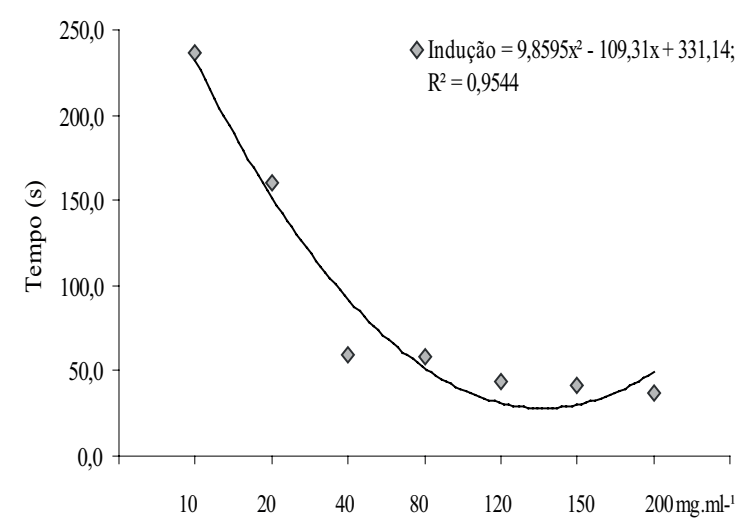

Fonte: Elaboração dos autores.

A utilização do eugenol como anestésico para peixes vem sendo testada para diferentes espécies. Gonçalves et al., (2008) observaram para juvenis de pacu (Piaractus mesopotamicus) que o eugenol é eficiente como anestésico na dose de $50 \mathrm{mg} \mathrm{L^{-1 }}$. A utilização deste anestésico para matrinxã (Brycon amazonicus) é eficiente nas concentrações entre 40 a $50 \mathrm{mg} \mathrm{L}^{-1}$ (INOUE; SANTOS NETO; MORAES, 2003) e para a rápida indução anestésica para tilápia (Oreochromis niloticus) recomenda-se utilizar 75mg.L-1 (VIDAL et al., 2008).

Os valores plasmáticas de glicose apresentaram elevação significativa em resposta ao banho
B

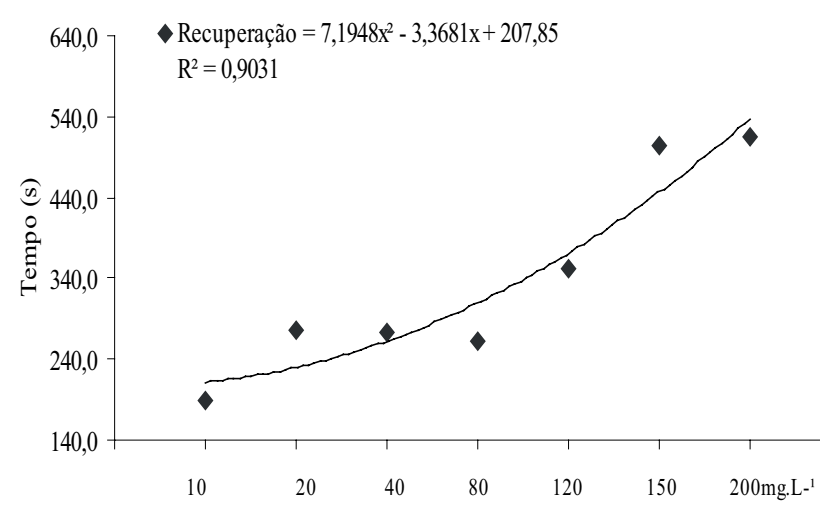

anestésico com eugenol (Figura 2). Segundo Zahl, Samuelsen e Kiessling, (2012) o uso de anestésico pode ter efeitos secundários indesejados que reduzem o bem-estar dos peixes, como a acidose. O efeito dos anestésicos, como redutor de estresse em peixes, é controverso, uma vez que respostas como glicemia vem sendo observado nesta situação (BARBOSA; MORAES; INOUE, 2007; INOUE et al., 2011). A glicemia, uma das respostas fisiológicas mais utilizadas como indicador de estresse em peixes, aumenta em resposta a algum fator estressante para suprir a maior demanda energética (MORGAN; IWAMA, 1997). 
Figura 2. Resposta plasmática de glicose de jundiá da Amazônia (Leiarius marmoratus) submetidos à diferentes concentrações de eugenol. Letras distintas reportam diferença estatística pelo teste de Tukey $(\mathrm{P}>0,05)$.

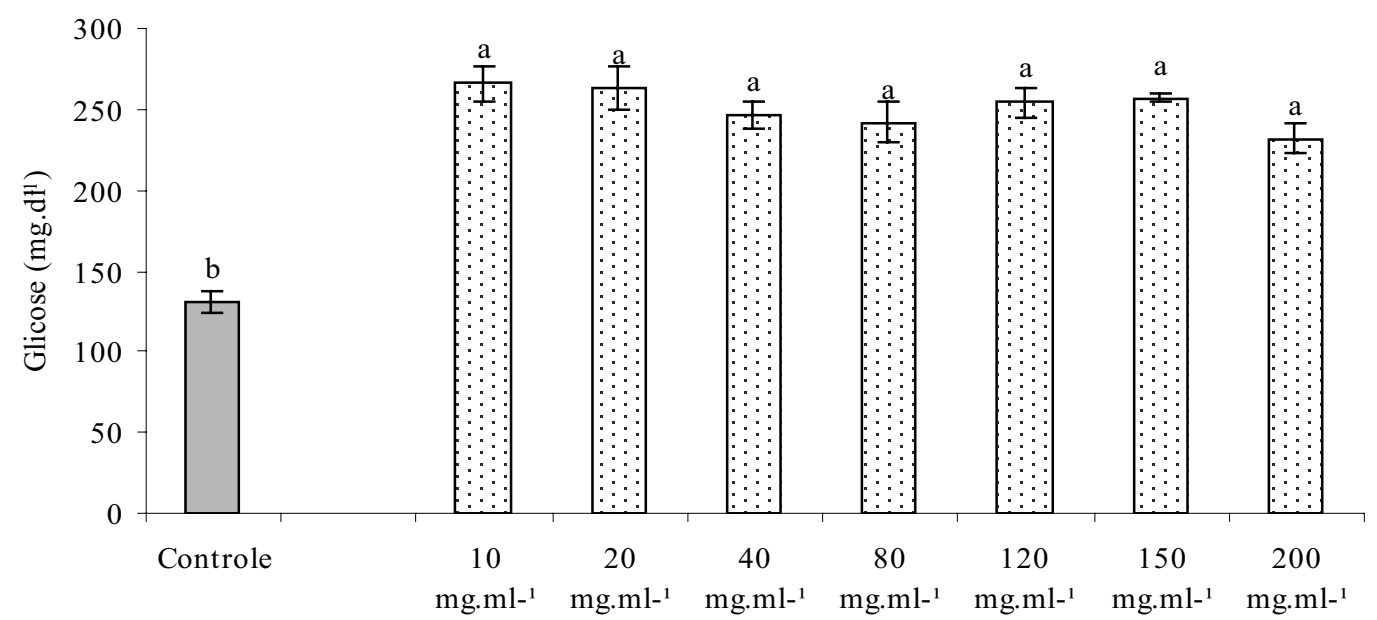

Fonte: Elaboração dos autores.

Segundo Inoue et al. (2011) a transferência dos peixes de local provoca estímulos adversos suficientes para iniciar as respostas metabólicas ao estresse, resultando em aumento da glicose do plasma. Esses aumentos são interpretados de forma que tão logo o cérebro detecte um ou mais estímulos adversos, dois eixos metabólicos são ativados: CPI (cérebro-pituitária-inter renais) e CSC (cérebro, células simpáticas de cromafins). No entanto, neste trabalho o grupo controle que também passou pelo mesmo manejo não apresentou elevação da glicose plasmática, podendo assim ser atribuído esta resposta ao banho anestésico.

Os jundiás da Amazônia (Leiarius marmoratus) submetidos às diferentes concentrações de eugenol não apresentaram alterações na pressão de oxigênio no sangue $\left(\mathrm{PaO}_{2}\right)$, pressão de dióxido de carbono $\left(\mathrm{PaCO}_{2}\right)$ e $\mathrm{pH}$. Os peixes quando submetidos a concentrações acima de $80 \mathrm{mg}$. $\mathrm{L}^{-1}$ apresentaram aumento de bicarbonato (Tabela 2).

Tabela 2. Valores médios e desvios-padrão dos parâmetros $\mathrm{pH}$, bicarbonato $\left(\mathrm{HCO}_{3}^{-}\right)$, pressão parcial de oxigênio $\left(\mathrm{PaO}_{2}\right)$ e dióxido de carbono $\left(\mathrm{PaCO}_{2}\right)$ de jundiás da Amazônia (Leiarius marmoratus) submetidos a diferentes concentrações de eugenol.

\begin{tabular}{|c|c|c|c|c|}
\hline & pH & $\mathrm{HCO}_{3}^{-}$ & $\mathrm{PaO}_{2}$ & $\mathrm{PaCO}_{2}$ \\
\hline Controle & $7,23 \pm 0,05$ & $3,90 \pm 0,05 d$ & $84,90 \pm 7,1$ & $55,90 \pm 3,5$ \\
\hline \multicolumn{5}{|c|}{ Concentração (mg.L $\left.{ }^{-1}\right)$} \\
\hline 10 & $7,30 \pm 0,03$ & $4,20 \pm 0,04 \mathrm{c}$ & $76,90 \pm 2,1$ & $50,80 \pm 4,4$ \\
\hline 20 & $7,23 \pm 0,05$ & $4,30 \pm 0,09 \mathrm{c}$ & $76,20 \pm 5,4$ & $55,50 \pm 2,9$ \\
\hline 40 & $7,07 \pm 0,02$ & $4,39 \pm 0,01 \mathrm{c}$ & $76,30 \pm 4,4$ & $60,70 \pm 2,1$ \\
\hline 80 & $6,97 \pm 0,04$ & $5,19 \pm 0,06 b$ & $73,60 \pm 3,1$ & $52,00 \pm 7,6$ \\
\hline 120 & $7,13 \pm 0,03$ & $5,10 \pm 0,03 b$ & $73,07 \pm 5,4$ & $55,80 \pm 3,7$ \\
\hline 150 & $7,21 \pm 0,01$ & $6,40 \pm 0,02 \mathrm{a}$ & $66,30 \pm 2,0$ & $56,20 \pm 2,5$ \\
\hline 200 & $7,09 \pm 0,03$ & $6,30 \pm 0,01 \mathrm{a}$ & $63,10 \pm 2,2$ & $54,50 \pm 1,1$ \\
\hline
\end{tabular}

Letras distintas reportam diferença estatística pelo teste de Tukey $(\mathrm{P}>0,05)$. A ausência de letras nas colunas indica que não houve diferença estatística.

Fonte: Elaboração dos autores. 
A difusão das trocas gasosas nas brânquias dos teleósteos é mais dificultada pela excreção de $\mathrm{CO}_{2}$ do que pela captação de $\mathrm{O}_{2}$. A habilidade do sangue lamelar realizar o equilíbrio com a água é influenciada por muitos fatores como a alta solubilidade do $\mathrm{CO}_{2}$ no sangue, a dependência da excreção de $\mathrm{CO}_{2}$ e afinidade com a hemoglobina, a conversão de $\mathrm{HCO}_{3}$ em $\mathrm{CO}_{2}$ durante a passagem do sangue pela circulação branquial (LIMA BOIJINK et al., 2010).

Ressalta-se que os valores médios de $\mathrm{PaO}_{2}$ e $\mathrm{PaCO}_{2}$ não diferiram entre os grupos, apontando que o eugenol não provocou efeito sobre a depressão respiratória. Deve-se destacar que, que tais achados são condizentes com a ocorrência de hiperventilação, característico do estagio III de peixes anestesiados, pois os valores de $\mathrm{PaO}_{2}$ foram mantidos em níveis adequados, o que deve ter mantido suprimento adequado de oxigênio aos tecidos. As pressões de gases sanguíneos $\left(\mathrm{PaO}_{2}\right.$ e $\mathrm{PaCO}_{2}$ ) tendem a apresentar poucas variações na maioria das situações uma vez que o primeiro mecanismo de homeostasia está relacionado com aumento da freqüência cardíaca (KEEN; GAMPERL, 2012).

A hiperventilação poderia ser resultado do decréscimo na concentração de oxigênio no sangue (hipoxemia) induzida pela acidose (KEEN; GAMPERL, 2012). A elevação na concentração de $\mathrm{CO}_{2}$ no sangue é capaz de desencadear respostas cardiorrespiratórias diretamente via interação quimiorreceptores específicos de $\mathrm{CO}_{2} / \mathrm{pH}$, independente da concentração de $\mathrm{O}_{2}$ (PORTEUS; BRINK; MILSOM, 2012).

Neste estudo, não foi possível observar aumento das taxas da $\mathrm{PaCO}_{2}$ e concomitantemente, redução do $\mathrm{pH}$ durante a anestesia pelo eugenol o que caracterizaria um quadro de acidose respiratória. No entanto, observa-se que os maiores concentrações de eugenol (150 e $200 \mathrm{mg} . \mathrm{L}^{-1}$ ) proporcionaram um leve aumento nos níveis de bicarbonato $\left(\mathrm{HCO}_{3}^{-}\right) \mathrm{O}$ que supostamente compensou a acidose, mantendo os valores de $\mathrm{pH}$ próximos a neutralidade. Sabese que o aumento na pressão de $\mathrm{CO}_{2}$ arterial, e concomitante diminuição do $\mathrm{pH}$, são inevitáveis durante a hipercarbia, mecanismo este utilizado para alterar o gradiente de difusão entre o sangue e água diminuindo a $\mathrm{PCO}_{2}$ (MILSON, 2012).

As concentrações de sódio, cloreto, potássio e cálcio plasmático mantiveram-se praticamente constantes (Tabela 3), o que indica que os procedimentos experimentais aparentemente não tiveram intensidade nem duração suficientes para induzir alterações no balanço eletrolítico do jundiá da Amazônia. O equilíbrio de sais no plasma pode ser alterado em resposta às mudanças da quantidade de água no sangue que passa pelas brânquias, alterando, também, as trocas de íons entre os meios interno e externo (MCDONALD; MILLIGAN, 1997). O estresse de manejo provoca alterações nos níveis de sódio, cloreto, cálcio e potássio de truta arco-íris Oncorhynchus mykiss (TAHMASEBIKOHYANI et al., 2012). No entanto, a utilização de eugenol em matrinxã (Brycon amazonicus) também não proporcionou mudanças no balanço hidroeletrilítico (BARBOSA; MORAES; INOUE, 2007).

O eugenol é um anestésico adequado para juvenis de jundiá da Amazônia (Leiarius marmoratus). Este produto pode ser utilizado em concentrações de $40 \mathrm{mg}$. L ${ }^{-1}$ sem provocar alterações hemogasométricas. 
Tabela 3. Valores plasmáticos de sódio, cloreto, potássio e cálcio de jundiá da Amazônia (Leiarius marmoratus) submetidos à diferentes concentrações de eugenol.

\begin{tabular}{ccccc}
\hline & \multicolumn{4}{c}{ Íons plasmáticos (nmol.L ${ }^{-1}$ ) } \\
\cline { 2 - 5 } & Sódio & Cálcio & Cloreto & Potássio \\
\hline Controle & $139,90 \pm 3,8$ & $1,11 \pm 0,8$ & $109,40 \pm 8,3$ & $1,56 \pm 0,3$ \\
Concentração $\left(\right.$ mg. $\left.^{-1}\right)$ & & & & \\
$\mathbf{1 0}$ & $128,80 \pm 4,2$ & $1,69 \pm 0,1$ & $105,10 \pm 8,2$ & $5,47 \pm 0,2$ \\
$\mathbf{2 0}$ & $137,70 \pm 2,7$ & $1,32 \pm 0,4$ & $107,60 \pm 1,4$ & $4,22 \pm 0,1$ \\
$\mathbf{4 0}$ & $127,00 \pm 3,4$ & $0,97 \pm 0,3$ & $99,00 \pm 4,2$ & $4,26 \pm 0,3$ \\
$\mathbf{8 0}$ & $142,60 \pm 5,9$ & $0,96 \pm 0,9$ & $110,60 \pm 0,3$ & $4,22 \pm 0,8$ \\
$\mathbf{1 2 0}$ & $131,70 \pm 7,6$ & $0,75 \pm 0,3$ & $102,00 \pm 3,0$ & $2,63 \pm 0,1$ \\
$\mathbf{1 5 0}$ & $131,90 \pm 4,5$ & $1,03 \pm 0,4$ & $103,90 \pm 4,1$ & $4,30 \pm 0,1$ \\
$\mathbf{2 0 0}$ & $133,20 \pm 3,3$ & $0,93 \pm 0,8$ & $104,50 \pm 8,3$ & $2,42 \pm 0,2$ \\
\hline
\end{tabular}

Letras distintas reportam diferença estatística pelo teste de Tukey $(\mathrm{P}>0,05)$. A ausência de letras nas colunas indica que não houve diferença estatística.

Fonte: Elaboração dos autores.

\section{Agradecimento}

A piscicultura Douradense Ltda. por disponibilizar os peixes para o ensaio. A Dra. Fernanda P. L. Zauith Diretora do Hospital Veterinário da UNIGRAN.

O estudo foi aprovado pela comissão de bioética e foi realizado de acordo com as normas técnicas de biosegurança e ética. Processo 104/11

\section{Referências}

BARBOSA, L. M.; MORAES, G.; INOUE, L. A. K. A. Metabolic responses of matrinxa to eugenol in anesthetic baths. Acta Scientiarum Biological Sciences, Maringá, v. 29, n. 3, p. 255-260, 2007.

DELBON, M. C. E.; PAIVA, M. J. T. R. Eugenol em juvenis de tilápia do Nilo: concentrações e administrações sucessivas. Boletim do Instituto de Pesca, São Paulo, v. 38, n. 1, p. 43-52, 2012.

DIEMER, O.; NEU, D. H.; BITTENCOURT, F.; SIGNOR, A.; BOSCOLO, W. R.; FEIDEN, A. Eugenol como anestésico para jundiá (Rhamdia voulezi) em diferentes pesos. Semina: Ciências Agrárias, Londrina, v. 33, n. 4, p. 1495-1500, 2012.

DINIZ, N. M.; HONORATO, C. A. Algumas alternativas para diminuir os efeitos do estresse em peixes de cultivo - revisão. Arquivo de Ciências Veterinária e Zoologia UNIPAR, Umuarama, v. 15, n. 2, p. 149-154, 2012.
EVANS, D. H.; PIERMARINI, P. M.; CHOE, K. P. The Multifunctional fish gill: dominant site of gas exchange, osmoregulation, acid-base regulation, and excretion of nitrogenous waste. Physiology Review, Bethesda, v. 85, n. 1, p. 97-177, 2005.

GIMBO, R.Y.; SAITA, M.; GONÇALVES, A.; TAKAHASHI, L. Diferentes Concentrações de benzocaína na indução anestésica do lambari do rabo amarelo (Astyanax altiparanae). Revista Brasileira de Saúde e Produção Animal, Salvador, v. 9, n. 2, p. 350.357, 2008.

GONÇALVES, A. F. N.; SANTOS, E. C.; FERNANDES, J. B. K.; TAKAHASHI, L. S. Mentol e eugenol como substitutos da benzocaína na indução anestésica de juvenis de pacu. Acta Scientiarum Animal Sciences, Maringá, v. 30, n. 3, p. 339-344, 2008.

INOUE, L. A. K.; BOIJINK, C. L.; RIBEIRO, P. T.; SILVA, A. M. D.; AFFONSO, E. G. Avaliação de respostas metabólicas do tambaqui exposto ao eugenol em banhos anestésicos. Acta Amazonica, Manaus, v. 41, n. 2, p. 327-332, 2011.

INOUE, L. A. K. A.; HACKBARTH, A.; MORAES, G. Assessment of 2-phenoxyethanol and benzocaine as anesthetics for field procedures in matrinxa (Brycon cephalus). Biodiversidade Pampeana, Uruguaiana, v. 2, n. 1, p. 10-15, 2004.

INOUE, L. A. K. A.; SANTOS NETO, C.; MORAES, G. Clove oil as anesthetic for juveniles of matrinxã Brycon cephalus (Gunther, 1869). Ciência Rural, Santa Maria, v. 33, n. 5, p. 943-947, 2003. 
KANANI, H. G.; AHADIZADEH, S. Use of propofol as an anesthetic and its efficacy on some hematological values of ornamental fish Carassius auratus. Springer Plus, Cham, v. 2, n. 76, p. 1-5, 2013.

KEEN, A.N. ; GAMPERL, A. K. Blood oxygenation and cardiorespiratory function in steelhead trout (Oncorhynchus mykiss) challenged with an acute temperature increase and zatebradine-induced bradycardia. Journal of Thermal Biology, New York, v. 37, n. 3, p. 201-210, 2012.

LIMA BOIJINK, C.; FLORINDO, L. H.; LEITE, C. A. C.; KALININ, A. L.; MILSOM, W. K.; RANTIN, F. T. Hypercarbic cardiorespiratory reflexes in the facultative air-breathing fish jeju (Hoplerythrinus unitaeniatus): the role of branchial $\mathrm{CO}_{2}$ chemoreceptors. Journal of Experimental Biology, London, v. 213, p. 2797-2807, 2010.

MAZZAFERA, P. Efeito alelopático do extrato alcoólico do cravo-da-índia e eugenol. Revista Brasileira de Botânica, São Paulo, v. 26, n. 2, p. 231-238, 2003.

McDONALD, G.; MILLIGAN, L. Ionic, osmotic and acid-base regulation in stress. In: IWAMA, G. K. et al. (Ed.). Fish stress and health in aquaculture. Cambrigde: Cambridge University Press, 1997. p. 119-144.

MILSOM, W. K. New insights into gill chemoreception: receptor distribution and roles in water and air breathing fish. Respiratory Physiology \& Neurobiology, Amsterdam, v. 184, n. 3, p.326-339, 2012.

MORGAN, J. D.; IWAMA, G. K. Measurements of stressed states in the field. In: IWANA, G. W.; PICKERING, A. D.; SUMPTER, J. P. (Ed.). Fish stress and health in aquaculture. Cambridge: University Press, 1997. p. 247-270.

OKAMOTO, M. H.; TESSER, M. B.; LOUZADA, L. R.; SANTOS, R. A.; SAMPAIO, L. A. Benzocaína e eugenol como anestésicos para juvenis do pampo Trachinotus marginatus. Ciência Rural, Santa Maria, v. 39, n. 3, p. 866-870, 2009.

PADUA, S. B.; PIETRO, P. S.; IGLESSIAS-FILHO, P. S.; ISHIKAWA, M. M.; HISANO, H. Mentol como anestésico para dourado (Salminus brasiliensis). Boletim do Instituto de Pesca, São Paulo, v. 36, n. 2, p. 143-148, 2010.

PARK, M. O.; HUR, W. J.; IM, S. T.; SEOL, D. W.; LEE, J.; PARK, I. S. Anaesthetic efficacy and physiological responses to clove oil anaesthetized kelp grouper Epinephelus bruneus. Aquaculture Research, Oxford, v. 39, n. 8, p. 877-884, 2008.

PEREIRA-DA-SILVA, E. M.; OLIVEIRA, R. H. F.; RIBEIRO, M. A. R.; COPPOLA. M. P. Efeito anestésico do óleo de cravo em alevinos de lambari. Ciência Rural, Santa Maria, v. 39, n. 6, p. 1851-1856, 2009.

PORTEUS, C. S.; BRINK, D. L.; MILSOM, W. K. Neurotransmitter profiles in fish gills: putative gill oxygen chemoreceptors. Respiratory Physiology \& Neurobiology, Amsterdam, v. 184, n. 3, p. 316-325, 2012.

ROSS, L. G.; ROSS, B. Anaesthetic and sedative techniques for aquatic animals. 3. ed. Oxford: Blackwell, 2008. $222 \mathrm{p}$.

ROTILI, D. A.; DEVENS, M. A.; DIEMER, O.; LORENZ, E. K.; LAZZARI, R.; BOSCOLO, W. R. Uso de eugenol como anestésico em pacu. Pesquisa Agropecuária Tropical, Goiânia, v. 42, n. 3, p. 288-294, 2012.

SÁNCHEZ, J. M.; MOYETONES, F.; CERDÁ, M. J. Influencia del contenido proteico en el crecimiento de alevines de bagre yaque, Leiarius marmoratus, alimentados con concentrados comerciales. Zootecnia Tropical, Maracay, v. 27, n. 2, p. 187-194, 2009.

SON, MAENG-HYUN; PARK, MIN-WOO; MYEONG, JEONG-IN; KIM, DAE-JUNG; KIM, BYOUNG-HAK; JO, Q-TAE; JEON, IM-GI. Anaesthetic tolerance of juvenile black rockfish Sebastes schlegeli, produced for wild stock enhancement. Ocean and Polar Research, Korea, v. 23, n. 3, p. 285-290, 2001.

TAHMASEBI-KOHYANI, A.; KEYVANSHOKOOH, S.; NEMATOLLAHI, A.; MAHMOUDI, N.; PASHA-ZANOOSI, H. Effects of dietary nucleotides supplementation on rainbow trout (Oncorhynchus mykiss) performance and acute stress response. Fish Physiology and Biochemistry, Amsterdam, v. 38, n. 2, p. 431-440, 2012.

TRINDER, P. Determination of glucose in blood using glucose oxidase with an alternative oxygen acceptor. Analytical Clinical Biochemistry, London, v. 6, p. 24-27, 1969.

URBINATI, E. C.; GONÇALVES, F. D. Pacu (Piaractus mesopotamicus). In: BALDISSEROTO, B.; GOMES, L. C. (Ed.). Espécies nativas para piscicultura no Brasil. Santa Maria: UFSM, 2005. p. 225-246.

VIDAL, L. V. O.; ALBINATI, R. C. B.; ALBINATI, A. C. L.; LIRA, A. D.; ALMEIDA, T. R.; SANTOS, G. B. Eugenol como anestésico para a tilápia-do-nilo. Pesquisa Agropecuária Brasileira, Brasília, v. 43, n. 8, p. 10691074, 2008.

ZAHL, I.; SAMUELSEN, O.; KIESSLING, A. Anaesthesia of farmed fish: implications for welfare. Fish Physiology and Biochemistry, Amsterdam, v. 38, n. 1, p. 201-218, 2012. 
